\title{
Cambios nictemerales de variables físicas y químicas en la Ciénaga de Paticos, complejo cenagoso de Ayapel, Colombia
}

\author{
Yimmy Montoya M. ${ }^{1}$ \& Néstor Aguirre R..$^{1,2}$ \\ 1. Grupo de Investigación Gaia, Departamento de Ingeniería, Universidad de Antioquia, apartado 1226, Medellín, Cra \\ 55 Av. Del Ferrocarril, Colombia. Fax: 57-4-2196568, yimmymontoya3@hotmail.com \\ 2.nnaguirre@udea.edu.co
}

Recibido 18-VI-2008. Corregido 22-II-2009. Aceptado 25-III-2009.

\begin{abstract}
Nictemeral variation of physical and chemical variables in the Paticos wetland, Ayapel swamp complex, Colombia. The nictemeral variation of abiotic factors in a key factor for tropical organisms. We evaluated some climatic, physical and chemical variables during four surveys of the hydrologic cycle in the Paticos wetland $\left(8^{\circ} 21^{\prime} 08.2^{\prime \prime} \mathrm{N}, 75^{\circ} 08^{\prime} 45.7^{\prime} \mathrm{W}\right)$. Of climatic variables, pluviosity presented the highest variation $(\mathrm{C} . \mathrm{V}$. = $1022 \%$ ) followed by air temperature (C.V. $=19.7 \%)$. There was a high relation in the coefficients of variation for these variables (84:1). This relation may be associated with altitude. Throughout the day-night cycle, most variables presented significant differences; except for pluviosity, air and water temperature (because of their high variability). Variables most related with nictemeral variation were pluviosity, wind speed and direction, air temperature and dissolved oxygen. Rev. Biol. Trop. 57 (3): 635-646. Epub 2009 September 30.
\end{abstract}

Key words: tropical wetland, physical limnology, seasonal variation, nictemeral variation.

Pese a que en Colombia las ciénagas poseen un área de 478419 hectáreas (Arias 1985) y que es reconocida la importancia de las ciénagas desde muchas servicios ambientales (reguladores de los regímenes hidrológicos de las áreas tributarias, amortiguadores climáticos, fuente de aprovisionamiento de agua, medio de transporte acuático, oportunidades de turismo y recreación, retención de sedimentos y nutrientes y un alto valor biogénico), son sistemas poco investigados.

En los trópicos los estudios de las variaciones nictemerales de parámetros limnológicos, sobre todo en ambientes rasos son relevantes, siendo considerados por varios autores más importantes que las variaciones estacionales (Barbosa 1981, Esteves 1988, Gavilán 1990). El estudio de la variación nictemeral de la temperatura del agua, oxígeno disuelto y otras variables acopladas a medidas climatológicas tales como el viento, la radiación solar y la precipitación tiene gran importancia como indicadores del funcionamiento en cortos periodos de tiempo de los sistemas acuáticos. Las oscilaciones periódicas son debidas no solo a los ciclos de luz-oscuridad que afectan las actividades de los organismos, sino a la acción también de los factores climáticos sobre la estructura vertical del sistema.

A partir de estos ciclos y de acuerdo a la intensidad y la frecuencia de la entrada de energía turbulenta, se favorece la mezcla y el incremento en la diversidad de las comunidades acuáticas, como consecuencia del carácter intermedio de la perturbación. En los ambientes lacustres los procesos de mezcla dependen de las fuerzas externas como la entrada y salida de agua, corrientes de turbiedad, viento, flujo de calor superficial y la morfometría del sistema (Gocke et al. 1981).

Las fluctuaciones limnológicas en un periodo de 24 horas juegan un papel fundamental 
en la caracterización de los cuerpos de agua tropicales (Esteves et al. 1988). Esto porque determinan los procesos biológicos y bioquímicos que allí se desenvuelven, generalmente caracterizados por profundas alteraciones, no siempre previsibles.

En Colombia en sistemas lenticos se han realizado algunos trabajos sobre el tema (Ramírez 1995, Ramírez \& Cogollo 2004, Wills \& Benjumea 2004, Montoya 2008). Estos autores plantean la necesidad de realizar estudios nictemerales, ya que la elevada temperatura y radiación, relativamente constantes a lo largo de todo el año, ratifican la hipótesis de que los ecosistemas acuáticos tropicales se encuentran dominados más por los ciclos de 24 horas que por el ciclo anual.

Este trabajo evalúo la magnitud de las variaciones nictemerales de la temperatura del aire, dirección y velocidad del viento, temperatura del agua, conductividad eléctrica, $\mathrm{pH}$ y oxígeno disuelto, en una ciénaga ubicada en la vertiente caribe colombiana, en cuatro campañas de muestreo dentro de un ciclo hidrológico, con el fin de producir elementos de discusión que aporten al tema de la magnitud de la variabilidad nictemeral y temporal (estacional) en el trópico.

\section{MATERIALES Y MÉTODOS}

Descripción del área de estudio. $\mathrm{El}$ sistema cenagoso de Ayapel se ubica en la jurisdicción del municipio de Ayapel, en el departamento de Córdoba, en la planicie Atlántica del norte de Colombia. Forma parte del macrosistema de humedales y zonas anegables de la depresión Momposina. La cuenca de la ciénaga tiene un área de $1504 \mathrm{~km}^{2}$, situada entre los 20 y $150 \mathrm{msnm}$. El territorio se ubica en la zona de bosque húmedo tropical con temperaturas entre los 26 y $29{ }^{\circ} \mathrm{C}$ (Anónimo 1986). El régimen de precipitaciones fluctúa entre un periodo de lluvias (abril y noviembre) y de sequía (diciembre y marzo), con un promedio de pluviosidad que oscila entre los 2000 y $2500 \mathrm{~mm}$. Geográficamente, la zona se ubica entre las coordenadas $8^{\circ} 04^{\prime}-8^{\circ} 30^{\prime}$ de latitud norte y $74^{\circ} 84^{\prime}-75^{\circ} 20^{\prime}$ de longitud oeste, sobre la vertiente oriental del río San Jorge. La Ciénaga de Paticos hace parte del complejo cenagoso de Ayapel y se encuentra en la parte occidental media del complejo (Fig. 1), el punto de muestreo se localizó a los $8^{\circ} 21^{\prime} 08.2^{\prime \prime}$ $\mathrm{N}$ y $75^{\circ} 08^{\prime} 45.7^{\prime \prime} \mathrm{W}$.

El drenaje, en general es dendrítico y presenta longitudes variables, siendo la mayor la quebrada Quebradona con cerca de $12.5 \mathrm{~km}$. La quebrada Ayapel, que se encuentra al oeste de la cabecera municipal y alimenta la Ciénaga de Paticos, es una de las más afectadas por la contaminación antrópica (Chalarca et al. 2007).

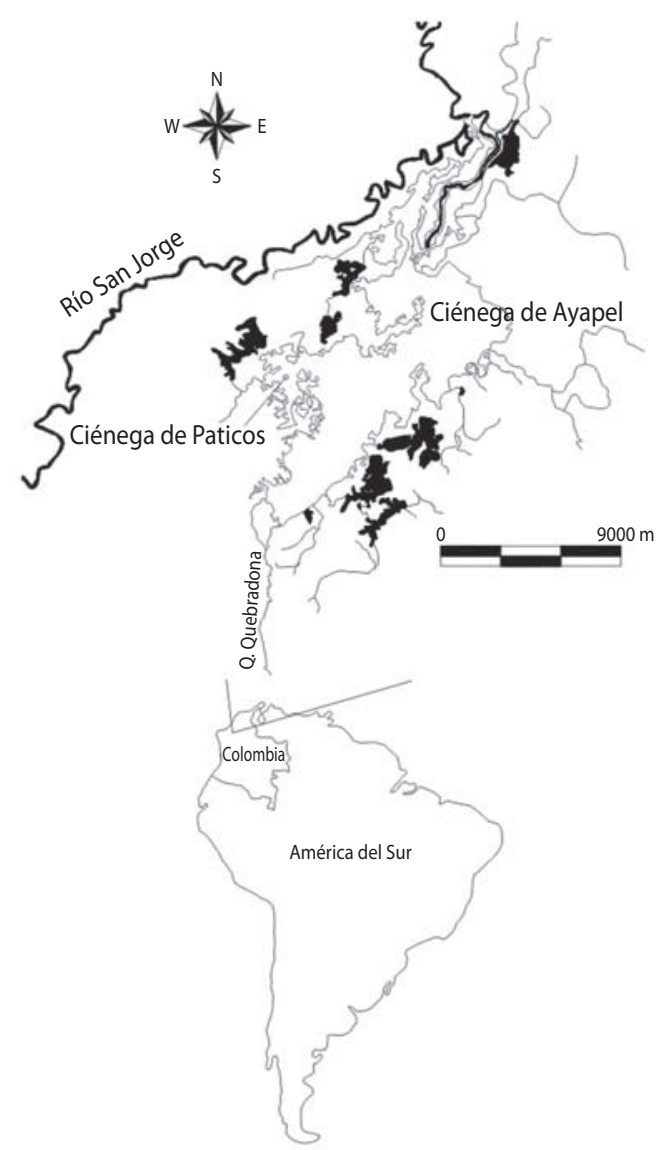

Fig. 1. Estación de muestreo en la Ciénaga de Paticos, complejo cenagoso de Ayapel, Colombia.

Fig. 1. Sampling station in the Paticos wetland, Ayapel wetland complex, Colombia. 
La oferta natural de la ciénaga y el paisaje circundante han sido objeto de explotación intensiva y desordenada, probablemente con efectos comprometedores respecto a su sostenibilidad. La pesca con métodos e intensidades inadecuadas al igual que el pastoreo de las franjas ribereñas y los vertimientos de las aguas residuales de la municipalidad, se constituyen en los principales factores de alteración natural.

Metodología. En el año 2006 en una estación de muestreo localizada en la zona central del espejo de agua de la Ciénaga de Paticos, se realizaron cuatro campañas nictemerales distribuidas durante el ciclo hidrológico del sistema (10-11 de mayo, 1-2 de agosto, 20-21 de septiembre y 4-5 de diciembre). Los registros fueron tomados cada dos horas comenzando a las 19:00 del primer día y terminando a la misma hora del día siguiente, para un total de 12 series de datos en cada campaña. En la columna de agua de aproximadamente $3 \mathrm{~m}$ se tomaron datos cada $10 \mathrm{~cm}$, desde subsuperficie hasta el fondo.

Para las lecturas en el campo de temperatura del agua, oxígeno disuelto, $\mathrm{pH}$ y conductividad eléctrica, se empleó una sonda multíparametro IQ. Los valores de pluviosidad, temperatura del aire, velocidad y dirección del viento fueron obtenidos mediante una estación meteorológica portátil Dawis Groundwater, con intervalos de cinco minutos durante los muestreos.

Los contrastes estadísticos de las variables se establecieron mediante un análisis de varianza de dos vías, de bloques aleatorios, modelo I. Los datos obtenidos fueron sometidos a análisis exploratorio mediante el uso de estadísticos de tendencia central (media aritmética, MA) y de dispersión (desviación estándar, S, y coeficiente de variación relativa de Pearson, C.V.). Para explicar la dependencia entre variables se efectúo un análisis de regresión y correlación lineal simple. Las pruebas estadísticas citadas fueron realizadas con en el paquete Statgraphics ${ }^{\circledR}$ plus v. 5.0.

\section{RESULTADOS}

El ciclo hidrológico es representado en la Fig. 2A, el cual muestra una distribución monomodal de las lluvias, con un máximo entre agosto y noviembre, y un periodo de sequía entre marzo y mayo. Esta característica pluviométrica se refleja en los niveles de la ciénaga (Fig. 2B). También puede observarse un aumento de los niveles de la ciénaga actuales, respecto a los valores promedios históricos.

El cuadro 1 presenta las características estadísticas básicas de la información nictemeral, se evidencia una mayor variación de

CUADRO 1

Principales medidas estadísticas de tendencia central

TABLE 1

Main statistical measurements of central tendency

$\begin{array}{lccccc}\text { Variable } & \text { Promedio } & \begin{array}{c}\text { Desviación } \\ \text { estándar }\end{array} & \text { Valor máximo } & \text { Valor mínimo } & \text { C.V. } \\ \text { Precipitación }(\mathrm{mm}) & 0.05 & 0.56 & 10.5 & 0 & 1022 \\ \text { Temperatura aire }\left({ }^{\circ} \mathrm{C}\right) & 29 & 3.52 & 35.3 & 22 & 12.1 \\ \text { Velocidad viento }(\mathrm{m} / \mathrm{s}) & 1.61 & 1.04 & 11.8 & 0 & 65 \\ \text { Dirección viento } & 9 & 5.4 & 16 & 0 & 59.9 \\ \text { Temperatura agua }\left({ }^{\circ} \mathrm{C}\right) & 29.7 & 1.29 & 33.2 & 26.3 & 4.3 \\ \text { Oxígeno disuelto }(\mathrm{mg} / \mathrm{l}) & 5.85 & 1.06 & 8.2 & 3.2 & 18.1 \\ \mathrm{pH}(\text { unidades de } \mathrm{pH}) & 7.02 & 0.15 & 7.8 & 6.61 & 2.2 \\ \text { Conductividad eléctrica }(\mu \mathrm{S} / \mathrm{cm}) & 56.2 & 21.9 & 334 & 6.2 & 39\end{array}$


A
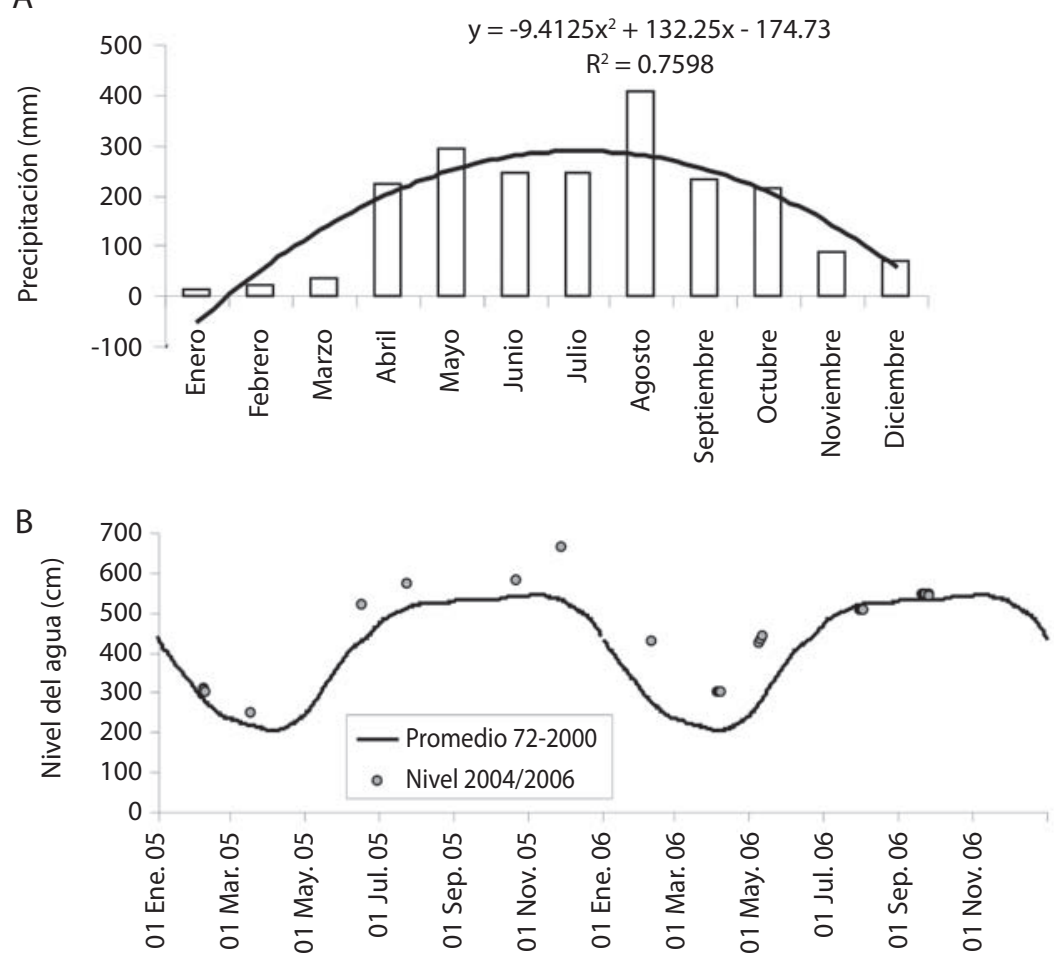

Fig. 2. A Variación de la pluviosidad mensual. B Variación de los niveles de la ciénaga.

Fig. 2. A Monthly variation of the rainfall. B Variation in water levels.

las variables climáticas que de las variables fisicoquímicas. Se encontró una variación alta de la precipitación pluvial (C.V. $=1022 \%$ ), las demás variables presentan variaciones bajas, excepto la velocidad y dirección del viento (C.V. $=65 \%$ y $59.9 \%$, respectivamente).

El cuadro 2 presenta los resultados del análisis de varianza de la información obtenida. Se evidencia que la mayoría de las variables evaluadas presentaron diferencias significativas ( $\mathrm{p}$ $<0.05$ ) excepto las variables ambientales y la temperatura del agua. Esta variable se presenta en la Fig. 3, la cual incluye los perfiles de temperatura y $\mathrm{pH}$ (excepto en el primer muestreo) en la columna de agua de la Ciénaga de Paticos,
CUADRO 2

Análisis estadísticos de las variables

TABLE 2

Statistical analysis of the variables

$\begin{array}{lcc}\text { Variable } & \mathrm{F} & \mathrm{p} \\ \text { Precipitación }(\mathrm{mm}) & 0.99 & 0.3313 \\ \text { Temperatura aire }\left({ }^{\circ} \mathrm{C}\right) & 0.21 & 0.6539 \\ \text { Velocidad viento }(\mathrm{m} / \mathrm{s}) & 8.16 & 0.0044 \\ \text { Dirección viento } & 8.14 & 0.0045 \\ \text { Temperatura agua }\left({ }^{\circ} \mathrm{C}\right) & 1.21 & 0.2707 \\ \text { Oxígeno disuelto }(\mathrm{mg} / \mathrm{l}) & 843.99 & 0.0000 \\ \text { pH }(\text { unidades de } \mathrm{pH}) & 280.29 & 0.0000 \\ \text { Conductividad eléctrica }(\mu \mathrm{S} / \mathrm{cm}) & 46.02 & 0.0000\end{array}$




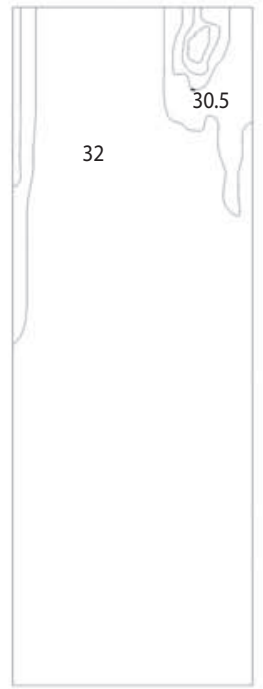

$\begin{array}{llll}7: 00 & 11: 00 & 3: 00 & 7: 00\end{array}$

Mayo

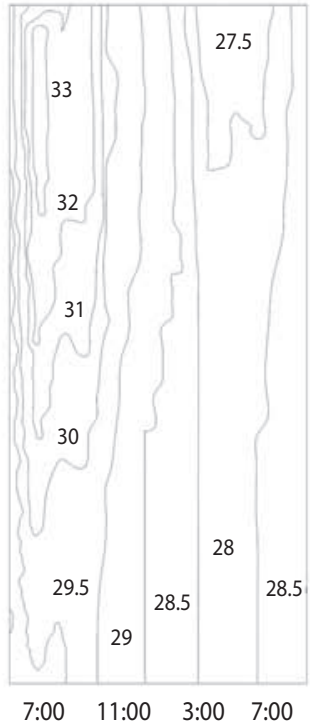

Agosto

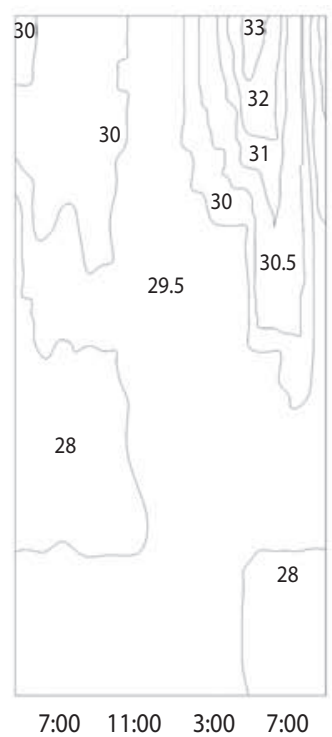

Septiembre

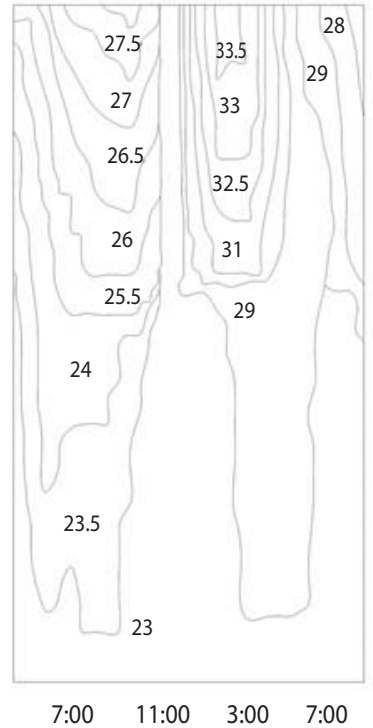

Diciembre

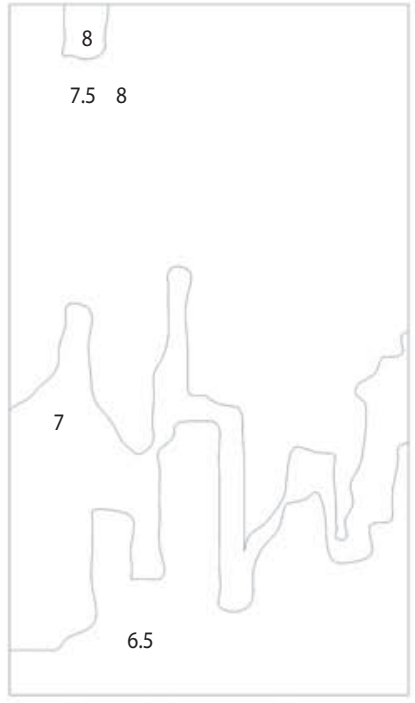

$\begin{array}{llll}7: 00 & 11: 00 & 3: 00 & 7: 00\end{array}$

Agosto

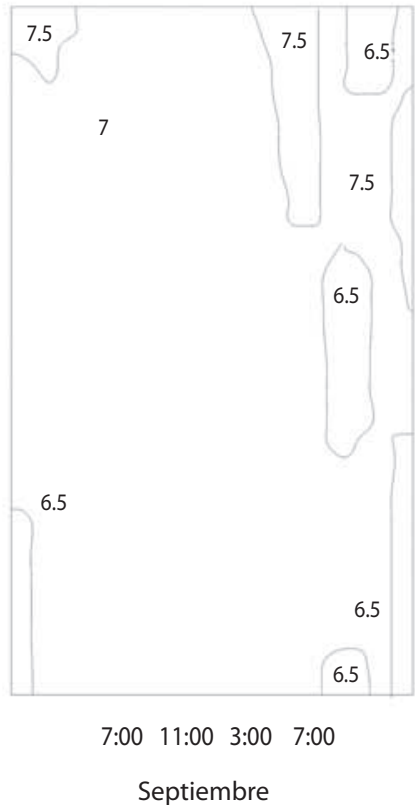

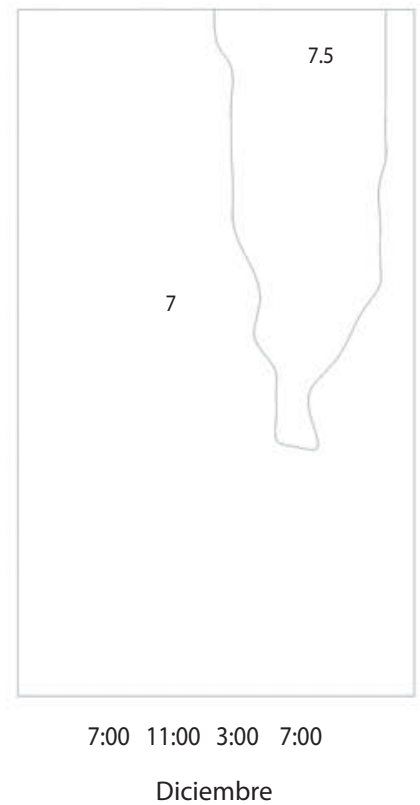

Fig. 3. Perfiles nictemerales de la temperatura del agua y del pH en la Ciénaga de Paticos.

Fig. 3. Nictemeral profiles of water temperature and $\mathrm{pH}$ in the Paticos wetland.

observándose alternancia entre los procesos de estratificación y mezcla.

La concentración de oxígeno presenta oscilaciones dinámicas a nivel nictemeral, aunque sin llegarse a presentar anoxia en la columna de agua (Fig. 4). La variación de la conductividad eléctrica está asociada a la mezcla y resuspensión de los materiales de fondo (Fig. 5). 

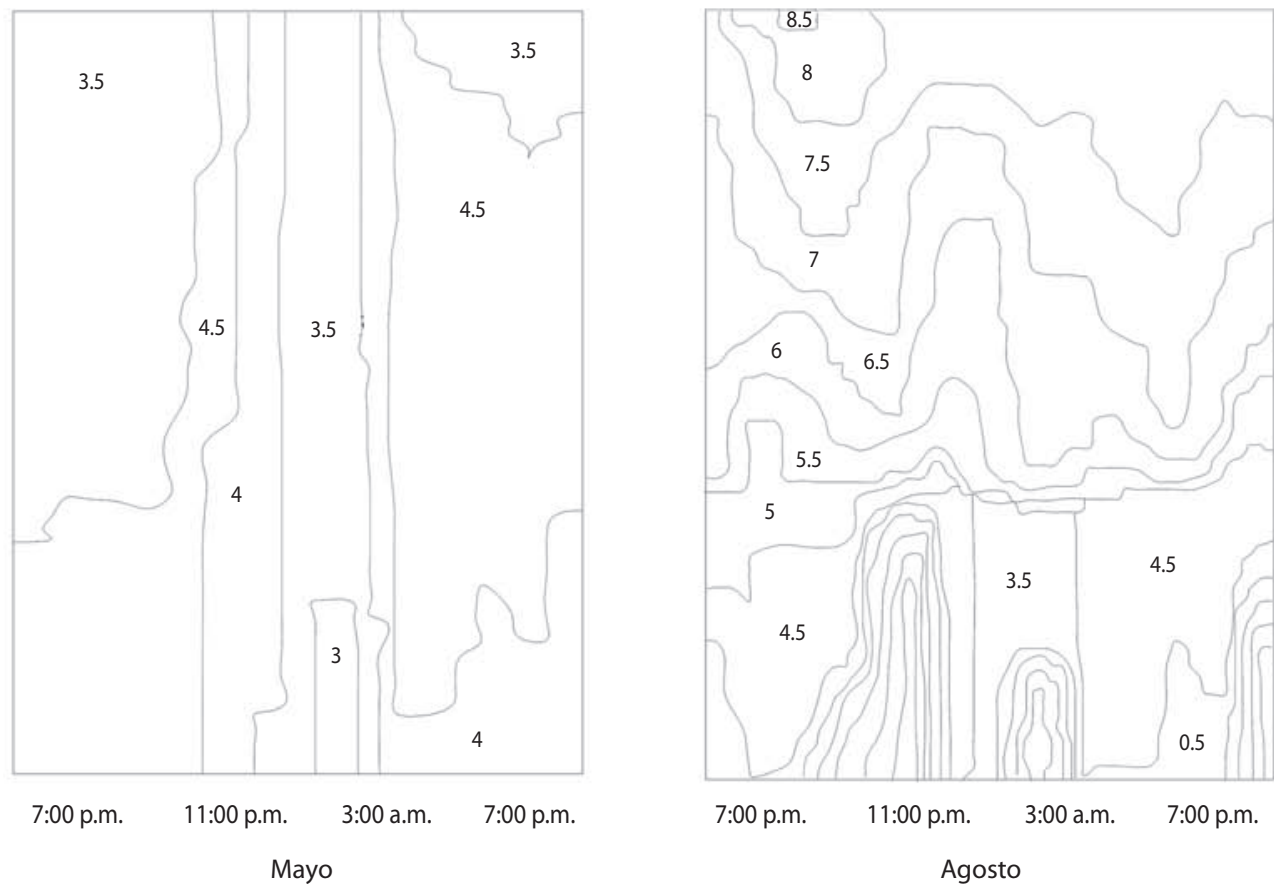

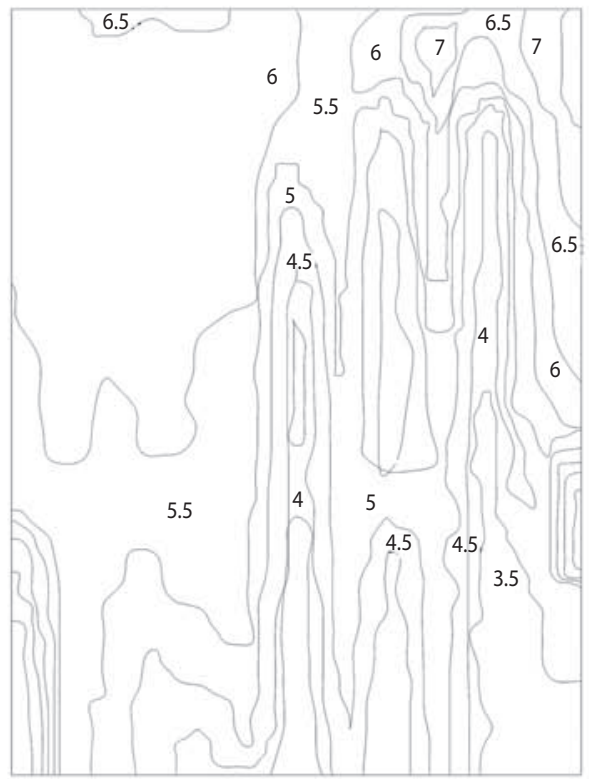

7:00 p.m. 11:00 p.m. 3:00 a.m. 7:00 p.m.

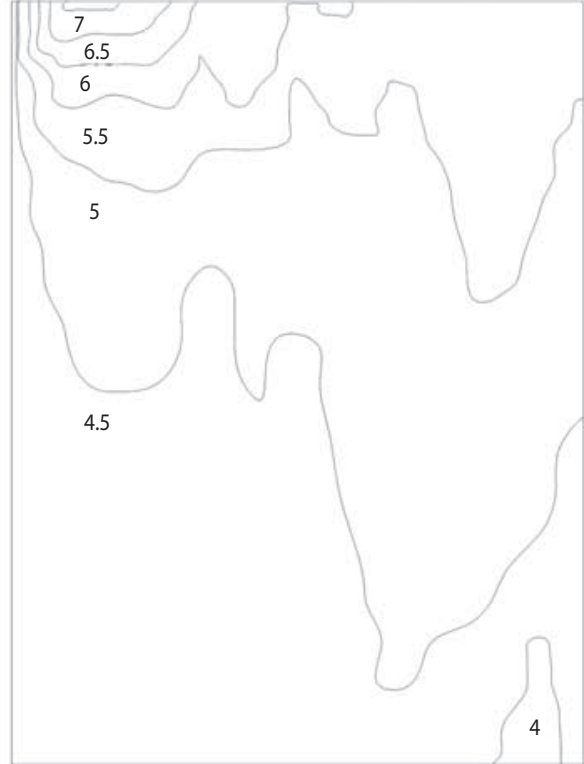

7:00 p.m. 11:00 p.m. 3:00 a.m. 7:00 p.m.

Setiembre

Diciembre

Fig. 4. Perfiles nictemerales de la concentración del oxígeno disuelto en la Ciénaga de Paticos.

Fig. 4. Nictemeral profiles of dissolved oxygen concentration in Paticos wetland. 


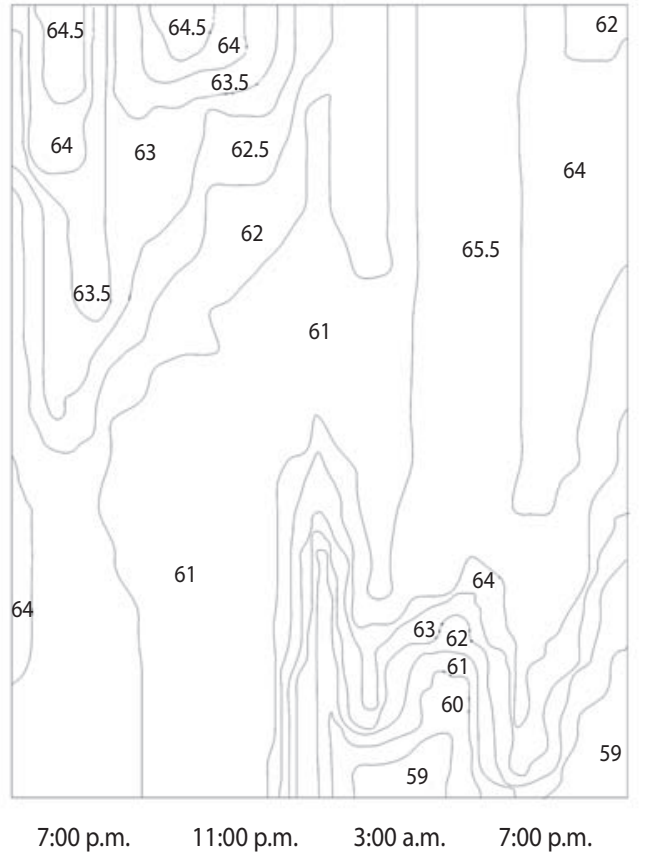

Agosto

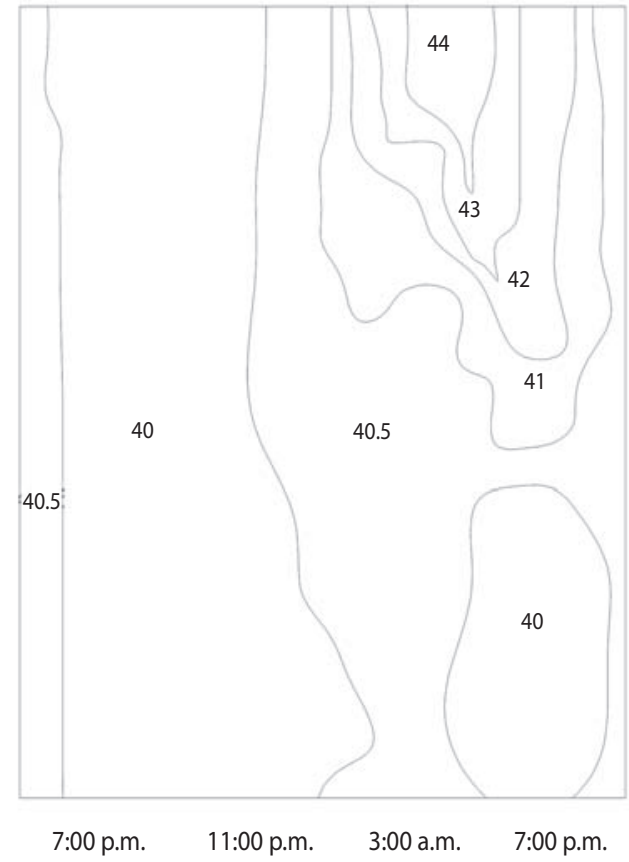

Septiembre

Fig. 5. Perfiles nictemerales de la conductividad eléctrica en la Ciénaga de Paticos.

Fig. 5. Nictemeral profiles of electrical conductivity in Paticos wetland.

Esta variable sólo se evaluó en dos muestreos (agosto y septiembre) ya que se presentaron problemas con la sonda multiparamétrica IQ, la misma situación se presento con el electrodo de $\mathrm{pH}$ en el primer muestreo (mayo).

La Ciénaga de Paticos es un sistema somero ya que su profundidad máxima fue inferior a $\operatorname{los} 10 \mathrm{~m}(\mathrm{Zmax}=3 \mathrm{~m})$, posee un amplio espejo de agua el cual permite intercambiar energía con su entorno haciendo de este un ecosistema dinámico, especialmente en el primer muestreo (mayo-aguas en ascenso) que corresponde al punto de menor columna de agua, se observa como al iniciar el muestreo nictemeral se presentó una estratificación térmica que alcanza los 1.5 m (50 \% de Zmax) entre las 19:00 y 21:00 horas, luego se presenta isotermia de la columna de agua hasta las 10:00 horas, hora a la cual el sol comenzó a calentar el espejo de agua, produciéndose una estratificación térmica que alcanzó el primer metro de la columna de agua (33 \% de Zmax). La resistencia termal relativa (RTR) permitió identificar la presencia una termoclina superficial la cual tiende a formarse después de las 11:00 am y a mezclarse alrededor de las 18:00 horas, por lo que la zona de mezcla tiende a aumentar entre el día y la noche, tomando valores entre $20 \mathrm{~cm}$ de profundidad y toda la columna de agua.

La concentración de oxígeno disuelto presentó un patrón similar, con máximos de concentración por debajo del termoclino $(1 \mathrm{~m})$, especialmente entre las 11:00 y las 18:00 horas, sin que se evidencien oxiclinas estables. La pluviosidad total durante el primer nictemeral fue de $21 \mathrm{~mm}$, se presentó una temperatura del aire promedio de $31.2{ }^{\circ} \mathrm{C}$. La velocidad del viento predominante fue de $1.61 \mathrm{~m} / \mathrm{s}$ en dirección oeste. 
Durante el segundo nictemeral (agosto) se presentaron aguas altas, este fue mayor pluviosidad (410 mm) del año 2006. Se evidenció estratificación térmica al comienzo del muestreo, la cual se rompió a las 14:00 horas debido al aumento de la nubosidad y de los vientos, permaneciendo la columna de agua mezclada durante el resto del muestreo y alcanzando a presentar una celda de menor temperatura a nivel subsuperficial debida a precipitaciones aisladas. Por otro lado, la concentración de oxígeno disuelto presentó los mayores valores respecto a los cuatro muestreos nictemerales, con un valor de $8.5 \mathrm{mg} / 1$ en un ambiente acuático que permaneció con la presencia de una oxiclina durante este periodo de investigación.

$\mathrm{El} \mathrm{pH}$ presentó también una estratificación definida durante todo el muestreo, con predominio de condiciones cercanas a la neutralidad en la mayor parte de la columna de agua y aguas ligeramente ácidas en la parte inferior de esta, debido a la resuspensión. La conductividad eléctrica registra también estratificación, aunque después de las 12:00 horas se evidenció una mezcla parcial de la columna de agua y resuspensión de materiales en el fondo de la ciénaga.

La pluviosidad total durante el segundo ciclo nictemeral fue de $27 \mathrm{~mm}$, se presentó una temperatura del aire promedio de $29.6{ }^{\circ} \mathrm{C}$. $\mathrm{La}$ velocidad del viento predominante fue de 1.89 $\mathrm{m} / \mathrm{s}$ en dirección $15^{\circ}$ sur-oeste.

El tercer nictemeral (septiembre) correspondió al periodo de lluvias en descenso con una pluviosidad mensual de $233 \mathrm{~mm}$. El perfil térmico presentó al igual que en el muestreo anterior estratificación definida al comienzo del muestreo y en las horas de la tarde del siguiente día, aunque se evidenció una fluctuación, ya que entre las 3:00 y las 8:00 horas se presentó una mezcla de casi toda la columna de agua. La concentración del oxígeno disuelto presentó estabilidad debido a la estratificación, especialmente en la primera parte del muestreo. Ya que después de las 5:00 hasta las 15:00 horas se presento un perfil difuso, en el que se notó mezcla parcial con valores fluctuantes de oxígeno disuelto.
A diferencia del perfil térmico y del oxígeno disuelto, la variación del $\mathrm{pH}$ durante este nictemeral fue mínima, se podría considerar la capa de agua como uniforme, salvo algunos leves incrementos en superficie y disminución parcial hacia el fondo.

La conductividad eléctrica presentó un patrón diferente al de otras variables mencionadas, es decir, las primeras 12 horas la tendencia del perfil fue ortogrado, después de las 7:00 horas se presenta estratificación química.

La pluviosidad total durante el tercer ciclo nictemeral fue de $0 \mathrm{~mm}$, se presentó una temperatura del aire promedio de $28.76{ }^{\circ} \mathrm{C}$. $\mathrm{La}$ velocidad del viento predominante fue de 1.7 $\mathrm{m} / \mathrm{s}$ en dirección oeste.

El cuarto nictemeral correspondió a aguas en descenso (diciembre), con una pluviosidad mensual de $71.3 \mathrm{~mm}$. El perfil térmico mostró estratificación durante casi las 24 horas con excepción de la media noche, tiempo en el cual por un corto lapso (1 hora) se presentó una columna isotérmica, registrándose temperaturas del agua del orden de los $23{ }^{\circ} \mathrm{C}$. Este episodio de mezcla generó una fluctuación en el oxígeno disuelto, el cual evidencia la disminución de la concentración con el aumento en la profundidad y presenta valores superiores a los $3.5 \mathrm{mg} / \mathrm{l}$. Además que, las isolíneas del oxígeno disuelto a media noche presentan un alargamiento en las diferentes capas del agua, generándose una perturbación parcial de la oxiclina. El pH mostró una columna uniforme inicialmente, excepto entre las 9:00 y las 17:00 horas, periodo en el cual se detectó un leve incremento del $\mathrm{pH}$ en el epilimnio y metalimnio.

La pluviosidad total durante el cuarto nictemeral fue de $9.1 \mathrm{~mm}$, se presentó una temperatura del aire promedio de $28.9^{\circ} \mathrm{C}$. La velocidad del viento predominante fue de $1.49 \mathrm{~m} / \mathrm{s}$ en dirección $5^{\circ}$ sur-oeste. La relación entre el coeficiente de variación de la pluviosidad y la temperatura del aire fue de $84: 1$, lo cual muestra el papel fundamental de la pluviosidad en la estacionalidad del sitio de muestreo. La Fig. 6 evidencia la tendencia que tiene esta relación a aumentar con la disminución en la altura sobre el nivel del mar. Esta figura fue elaborada 


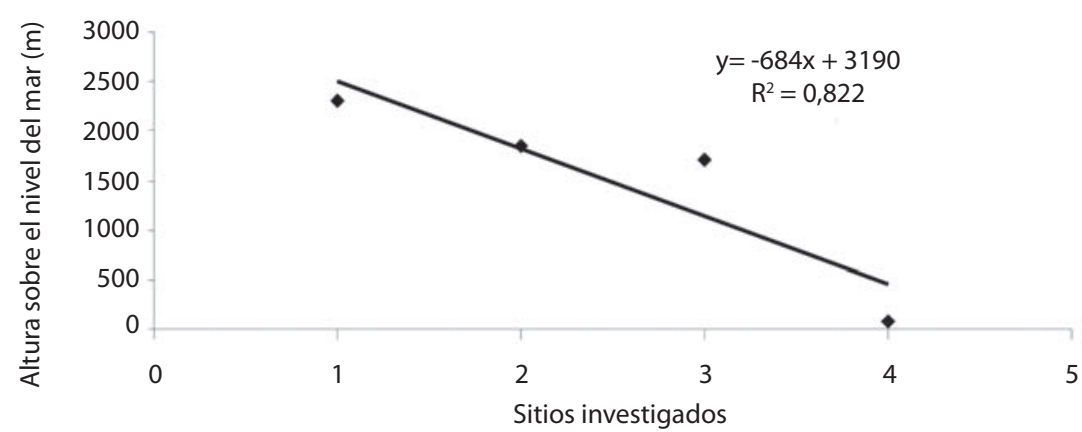

1. Río Medellín, 2. Laguna en Guatepé, 3. Lago Parque Norte, 4. Ciénaga Ayapel

Fig. 6. Relación entre los coeficientes de variación de la pluviosidad y la temperatura del aire.

Fig. 6. Relationship between coefficients of variation of rainfall and temperature.

a partir de la información de varias investigaciones realizadas en Colombia (Urrego \& Ramírez 2000, Ramírez 2000, Montoya 2008) y los datos registrados en esta investigación, lo que sugiere que los ecosistemas de zonas bajas tienen una mayor influencia de la precipitación, ya que la radiación presenta valores altos.

\section{DISCUSIÓN}

El gradiente vertical de la temperatura del agua presenta bajas amplitudes térmicas, ya que la mayor diferencia de esa variable favoreciendo la estratificación de la columna de agua fue de $1.1{ }^{\circ} \mathrm{C}(14: 00 \mathrm{~h}$, septiembre/2006) y la menor diferencia fue de $-1.05{ }^{\circ} \mathrm{C}(6: 00$ $\mathrm{h}$, diciembre/2006), lo cual indica un sobre enfriamiento de la capa superior de la columna de agua que antecede a la mezcla. Este hecho es característico en los lagos tropicales. Lewis (1987) considera que la identificación del epilimnio en un perfil vertical de temperatura en los lagos tropicales, es extremadamente difícil en función del gradiente vertical al ser típicamente pequeño.

El comportamiento térmico de esta ciénaga cambia de acuerdo al ciclo hidrológico, lo que contradice parcialmente la hipótesis de investigación, ya que la variabilidad nictemeral es mayor. En mayo las aguas de la Ciénaga de Paticos presentaron una estratificación superficial bien definida en las horas de mayor radiación solar, especialmente entre las 11:00 y las 19:00 horas, luego este gradiente disminuye debido al enfriamiento convencional de la superficie del agua. En agosto, se presenta mayor diferencia de temperatura entre las aguas superficiales y las profundas, por lo que la estratificación es más estable, especialmente durante las primeras ocho horas de muestreo. Luego, la presencia de lluvia en las horas de la tarde repercutió en la desestratificación de la columna de agua. La correlación entre la velocidad del viento y la precipitación permite inferir que este por ser el mes más lluvioso, presentó una influencia alta del viento sobre el perfil térmico de la ciénaga (cuadro 3).

El muestreo de septiembre evidencia un aumento de la fuerza de la estratificación a tal punto que esta se presenta durante la mayor parte del tiempo, aunque entre las 3:00 y 6:00 horas se alcanza una mezcla parcial de las capas de agua. Durante el muestreo de diciembre se observó alternancia entre mezcla-estratificación-mezcla, debido a que el muestreo inició en las horas de la noche (22:30 h) en donde se presentó inversión térmica, luego el gradiente térmico se acentuó formando termoclinos 
CUADRO 3

Regresión lineal entre las variables

TABLE 3

Linear regression among variables

$\begin{array}{lccc}\text { Variables relacionadas } & \text { Ecuación } & \mathrm{p} & \mathrm{r} \\ \text { Precipitación-Velocidad viento } & \mathrm{Y}=0,2541 \mathrm{x}-0,3544 & 0.0000 & 0,47 \\ \text { Dirección viento-Velocidad viento } & \mathrm{Y}=6,2947 \mathrm{x}+1,74 & 0.0000 & 0,33 \\ \text { Velocidad viento-Temperatura aire } & \mathrm{Y}=0,0355 \mathrm{x}+0,5805 & 0.0000 & 0,12 \\ \text { pH-Temperatura } & \mathrm{Y}=0,0383 \mathrm{x}+5,8745 & 0.0000 & 0,32 \\ \text { Oxígeno disuelto-Temperatura agua } & \mathrm{Y}=0,0174 \mathrm{x}+5,3281 & 0.0003 & 0,021\end{array}$

superficiales bien definidos, los cuales decrecen en altura hacia el final del día. Esto causa un aumento del enfriamiento convencional y al mismo tiempo, una disminución de la fuerza de flotación como resultado del aumento de la densidad en la capa superficial de agua. Esta mezcla diurna debida a los efectos del viento y la transferencia de calor a la atmósfera es un proceso bien conocido y típico de los lagos tropicales, porque la temperatura del agua muchas veces alcanza la temperatura del aire (Ramírez 2000).

Se puede concluir que la Ciénaga de Paticos es un ambiente cinético turbulento, altamente influenciado por los vientos, con estratificación diurna y mezcla nocturna (Fig. 7). El patrón térmico de este sistema puede clasificarse como polimíctico cálido continuo según la clasificación de Lewis (1987). La inestabilidad de la columna de agua puede llevar a la resuspensión de sedimentos de la ciénaga.

Los valores del $\mathrm{pH}$ en las aguas de la ciénaga fueron en su mayoría casi neutros, de 7 a 7.5 , durante el periodo lluvioso y con valores entre 6.5 a 7.5 en el periodo seco. En este lapso, los perfiles verticales presentaron resultados más homogéneos que en épocas de lluvia. Esta escasa variación tiene otra connotación a nivel biológico, la cual está asociada a la producción primaria planctónica y perifítica, que tienen poco peso en este sistema (Montoya \& Aguirre, en prep.), por lo que se puede considerar la ciénaga como un ecosistema heterotrófico subsidiado por los aportes alóctonos, lo que va en contravía al postulado común de que las ciénagas son sistema altamente productivos (Mitsch \& Gosselink 2000). Además, la reducida fluctuación del $\mathrm{pH}$ indica el poder tamponante de las aguas investigadas.

Esta investigación muestra que la mayor variación en las variables ambientales en ciclos nictemerales es debida a la fluctuación de la precipitación. En este trabajo al evaluar la fluctuación de esta variable cada cinco minutos durante los muestreos, permitió identificar una variación del $1022 \%$, siendo 15 veces superior a la fluctuación de la velocidad del viento, encontrándose correlación estadística entre ambas variables. Por otro lado, en esta investigación se reporta una relación entre los coeficientes de variación de la pluviosidad y la temperatura del aire casi tres veces mayor al ser comparado este cociente respecto a zonas de mayor altitud dentro del trópico, lo cual es un hecho interesante ya que puede verse como en los ecosistemas de tierras bajas la precipitación tiene un mayor peso en la introducción de variabilidad ambiental, introduciendo entropía a los sistemas alcanzando a generar patrones estacionales entre las épocas de lluvias y sequías.

La otra variable que presentó un coeficiente de variación alto fue la dirección del viento, este valor fue del $59.9 \%$, es decir, 17 veces inferior a la variación de la pluviosidad. Por otro lado, las variables evaluadas en el agua 

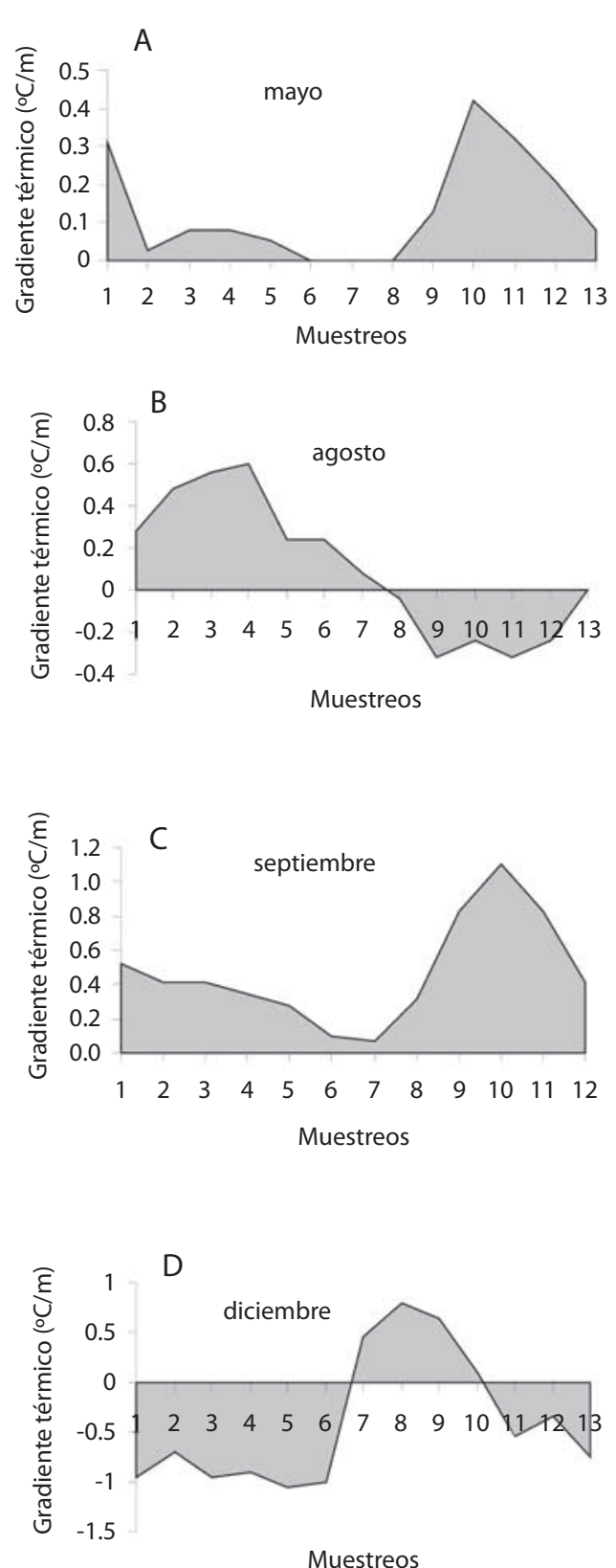

Fig. 7. Gradientes térmicos nictemerales en la Ciénaga de Paticos.

Fig. 7. Nictemeral thermal gradient in the Paticos wetland.

presentaron las menores variaciones, especialmente la temperatura del agua y el $\mathrm{pH}$, las cuales presentan variaciones menores al $5 \%$.
La conductividad eléctrica presentó mayores valores en el periodo seco que en el lluvioso, lo que posiblemente está asociado a la mezcla de la columna de agua y la resuspensión de material sestónico. El valor medio obtenido para esta variable $(56.2 \mu \mathrm{S} / \mathrm{cm}, \mathrm{C} . \mathrm{V} .=39 \%$, $\mathrm{n}=377)$ corresponde a una conductividad eléctrica de escala media, propia de un sistema mesotrófico. La conductividad eléctrica brinda información acerca de la magnitud de la producción primaria y de la descomposición, al igual que sobre la geoquímica de la cuenca circundante (Ramírez 2000), se puede considerar que los valores de escala media encontrados para esta variable al igual que su nivel de variación en el ciclo nictemeral están relacionados con una baja productividad primaria.

En la medida en la que disminuye la escala temporal de observación se aumenta la variabilidad de la información, lo que permite detectar fluctuaciones pequeñas de las variables climáticas, físicas y químicas las cuales repercuten en las comunidades biológicas y las variaciones de éstas afectan las variables físicas y químicas del agua. A nivel nictemeral todas las variables estudiadas presentaron variación estadística significativa, excepto la temperatura del aire y del agua y la pluviosidad, lo que puede implicar que su fluctuación es internalizada por lo componentes del sistema cenagoso, por lo que estas variables presentan baja fluctuación en ciclos cortos, pero al evaluarlas a través del ciclo hidrológico esta condición cambia.

En general, se encontró que la influencia del viento opera en relación con el periodo hidroclimático. Esta ciénaga se tipifica como polimíctica cálida continua, lo que asociado a su baja profundidad relativa, implica que se produzca resuspensión de materiales del fondo de la ciénaga, los cuales a su vez entran a jugar un papel activo en la dinámica de nutrientes del sistema.

En la Ciénaga de Paticos fueron observadas diferencias significativas entre los eventos de inestabilidad de la columna de agua y de estratificación en relación con la época del ciclo hidrológico y con la hora del muestreo, siendo esta última la que presentó la mayor variación. 


\section{AGRADECIMIENTOS}

Este estudio fue financiado por el convenio Universidad de Antioquia-GAIA-Universidad Nacional de Colombia, proyecto GreciaColciencias. Agradecemos la colaboración en las labores de campo de los miembros del grupo Gaia y a Alvaro Wills quién suministró la información meteorológica e hidrológica.

\section{RESUMEN}

Se evaluó la variación nictemeral de algunas variables climáticas, físicas y químicas durante cuatro muestreos dentro del ciclo hidrológico en la Ciénaga de Paticos ( $8^{\circ} 21^{\prime} 08.2^{\prime \prime} \mathrm{N}, 7^{\circ} 08^{\prime} 45.7^{\prime}$ W). Entre las variables climáticas, la pluviosidad presentó la mayor variación (C.V. $=1$ $022 \%$ ) seguida por la temperatura del aire (C.V. $=19.7 \%$ ). La elevada relación entre los coeficientes de variación de estas variables (84:1) está asociada a un incremento de esta relación con la disminución de la altura en la zona tropical. A través del ciclo día-noche la mayoría de las variables presentaron diferencias estadísticas significativas (excepto la pluviosidad, la temperatura del aire y del agua, lo cual se debe a su elevada variación). El análisis de correlación mostró que las variables más relacionadas con la variación nictemeral fueron la pluviosidad, la velocidad y dirección del viento, la temperatura del aire y del agua, el $\mathrm{pH}$ y la concentración de oxígeno disuelto.

Palabras clave: ciénaga tropical, limnología física, variación temporal, variaciones nictemerales.

\section{REFERENCIAS}

Anónimo. 1986. Estudio general de suelos de los municipios de Ayapel, Buena Vista, Planeta Rica y Pueblo Nuevo (Departamento de Córdoba). Instituto Geográfico Agustín Codazzi, Bogotá, Colombia. 282 p.

Anónimo. 2002. Plan básico de ordenamiento territorial. Municipio de Ayapel, Córdoba. PBOT, Montería, Córdoba, Colombia. 315 p.

Arias, P. 1985. Evaluación limnológica de las planicies inundables de la cuenca norte del río Magdalena. Proyecto para el desarrollo de la pesca continental. Inderena-FAO, Bogotá, Colombia. 35 p.

Barbosa, F.A. 1981. Variaçăo diurnal (24 hs) de parâmetros limnológicos básicos e da porductividade primaria do fitoplâncton da lagoa Carioca-Parque florestal do Rio Doc.e Disertacao Mestre, Universidae de Sâo Carlos, Sâo Carlos, Brasil. 356 p.

Chalarca, D., R. Mejía \& N. Aguirre. 2007. Aproximación a la determinación del impacto de los vertimientos de las aguas residuales domésticas del municipio de Ayapel, sobre la calidad del agua de la ciénaga. Rev. Fac. Ing. 40: 41-58.

Esteves, F.A., R.L. Bozelli, A.F. Camargo, F. Roland \& S.M. Thomaz. 1988. Variaçào diària (24 horas) de temperatura, $\mathrm{O}_{2}$ dossolvido, $\mathrm{pH}$ e alcalinidade em duas Lagoas costeiras do estado do Rio de Janeiro e sus implicaçóes no metabolismo destes ecosistemas. Acta Limnol. Bras. 11: 99-127.

Esteves, F.A. 1998. Fundamentos de limnología. Interciencia, Rio de Janeiro, Brasil. 176 p.

Gavilan, R.A. 1990. Flutuaçoes nictemerais dos fatores ecológicos na represa de Barra Bonita-Medio Tieté-S. P. Disertacao Mestre em Ciencias, Universidae de Sâo Carlos. Sâo Carlos, Brasil. 230 p.

Gocke, K., E. Laman, G. Rojas \& J. Romero. 1981. Morphometric and basic limnological data of Laguna Grande de Chirripó, Costa Rica. Rev. Biol. Trop. 29: 165-174.

Lewis, J. 1983. A revised classification of lakes based on mixing. Can. J. Fish Aquat. Sci. 40: 1779-1787.

Mitsch, W.J. \& J.G. Gosselink. 2000. Wetlands. John Wiley $\&$ Sons. New York, USA. 920 p.

Montoya-M, Y. 2008. Variaciones nictemerales de algunas variables climáticas, físicas y químicas, en una laguna somera en Guatapé-Antioquia, Colombia. Actual. Biol. 30: 83-96.

Ramírez, J.J. 1995. Variaciones verticales de temperatura y factores químicos en un ciclo de 24 horas en el embalse Punchiná, Antioquia, Colombia. Acta Limnol Bras. 7: 23-34.

Ramírez, J.J. 2000. Variación diurna y estacional del contenido calórico, la estabilidad y el trabajo del viento en una laguna tropical. Acta Limnol. Bras. 12: 39-54.

Ramírez, J.J. \& Y. Cogollo. 2004. Dinámica nictemeral y vertical de atributos físicos y químicos en tres ciénagas tropicales del parque nacional natural Los Katios, Chocó (Colombia). VI Seminario Colombiano de Limnología, Montería, Colombia. 124 p.

Urrego, A.P. \& J.J. Ramírez. 2000. Cambios diurnos en variables físicas y químicas en la zona de ritral del río Medellín, Colombia. Caldasia 22: 127-141.

Wills, A.T. \& C.A. Benjumea. 2004. Evaluación de la estratificación térmica y su relación con la hidrodinámica y la calidad del agua en la Ciénaga de Cachimbero. VI Seminario Colombiano de Limnología, Montería, Colombia. 124 p. 